\title{
Computer-aided synthesis of spherical and planar 4R linkages for four specified orientations
}

\author{
Guangming Wang ${ }^{1}$, Hao Zhang ${ }^{1}$, Xiaoyu $\mathrm{Li}^{1}$, Jiabo Wang ${ }^{2}$, Xiaohui Zhang ${ }^{1}$, and Guoqiang Fan ${ }^{1}$ \\ ${ }^{1}$ College of Mechanical and Electronic Engineering, Shandong Agricultural University, Tai' an, China \\ ${ }^{2}$ College of Engineering, Nanjing Agricultural University, Nanjing, China \\ Correspondence: Guangming Wang (gavinwang1986@163.com)
}

Received: 17 June 2018 - Revised: 25 February 2019 - Accepted: 23 May 2019 - Published: 25 June 2019

\begin{abstract}
According to the burmester theory, an infinite number of spherical or planar 4R linkages for a specific four-orientation task can be synthesized, but most of the linkage solutions calculated by this method are invalid because of motion defect, poor performance and others. In order to improve the synthesis efficiency, a program package based on Matlab is developed to find a satisfied linkage solution automatically and quickly. Firstly, the calculation on circle points and the center points based on the burmester theory in spherical problems is introduced. Secondly, the calculation methods of linkage defect discrimination, linkage type classification, linkage performance evaluation and solutions visualization based on the theory of spherical trigonometry are presented respectively. Thirdly, the synthesis calculation of program package is extended to the planar $4 \mathrm{R}$ linkage based on the theory of planar analytic geometry. Finally, the examples of the spherical synthesis problem and the planar synthesis problem based on solutions map are introduced to test the program package, the result proves this program package is effective and flexible.
\end{abstract}

\section{Introduction}

In this paper, the study work is to design a $4 \mathrm{R}$ linkage which can guide its coupler to pass four specified orientations smoothly and orderly. In order to accomplish this design task, graphing method and computer-aided synthesis method are used mainly at present. The theoretical basis of the graphing method is the theory of burmester, that is to say, connecting any two groups of center points and their corresponding circle points can obtain the desired linkage solution. Although the selection of center points or circle points are optional, majority of the linkage solutions have some problems such as circuit defect, branch defect, order defect or poor motion performance (Wang et al., 2010; Baskar and Bandyopadhyay, 2019a). For the linkage motion defect, the classical defect discrimination method is to study the segmentation technique of the burmester curve (Filemon, 1972; Waldron and Strong, 1978; Gupta and Beloiu, 1998). Considering the inefficiency of the graphing method and the inaccuracy of the discrimination of defective linkages, such as discarding some feasible solutions as uncertain solutions, its application have some limitations. With the development of computer-aided design, the burmester curve mentioned above can be obtained by programming with analytic geometry, but the existence of defective linkages makes it difficult to obtain feasible solutions. In order to get the most candidate solutions, one of the approaches is to allow some task variables to change in a certain range, so as to expand the number of solutions (Zhao et al., 2016; Baskar and Bandyopadhyay, 2019b; Chanekar et al., 2013; Sun et al., 2016). Although this method can increase the number of solutions, most of the increased linkage solutions still have motion defects, so it does not fundamentally eliminate the blindness of linkage synthesis process. Considering that modern computers can obtain massive solutions quickly and discriminate the defective linkage solutions in turn later (Myszka et al., 2009; Tipparthi and Larochelle, 2011; Bai, 2017), the method of type map (Han and Cao, 2019 ) is gradually applied in some linkage synthesis software, such as Synthetic (Su and McCarthy, 2005), Lincages (Erdman and Loftness, 2005), Sphinx (the latest version is SphinxPC) (Ruth and McCarthy, 1999; McCarthy, 2013) and the software developed by Han et al. (2013). Among them, specially, Sphinx is the first software to solve the spherical 
problems. The method of type map maps all the linkage solutions obtained by computer into a two-dimensional coordinate system and marks different linkage types with different colors. At the same time, the defective linkage solutions can be hidden. The blindness of linkage synthesis can be reduced by using the type map based on these synthesis software, but there are still some shortcomings: firstly, the biggest problem is that it is not flexible enough and must be designed the linkage according to the process set by the software; secondly, few software besides Sphinx have the synthesis ability of spherical linkage; finally, the type map method can only express the solutions distribution with different linkage type in the solution domain, however, in practical synthesis problems, the transmission performance and installation position of linkage solutions are more important than linkage types (Mendoza-Trejo et al., 2015). In order to make up for the shortcomings of the graphing method and computer-aided synthesis method mentioned above, a series of flexible functions for the synthesis, analysis and plotting of linkages are constructed under Matlab. These functions can be used for the synthesis calculation of spherical and planar 4R linkages. Compared with the previous studies, the main work of this study is as follows: the existing color-coded type map method is improved to express not only the discrete attribute of linkage type, but also the continuous attribute such as transmission performance; a defect discrimination method based on geometric method is proposed, and a pyramid structure of the circuit, branch and order defect discrimination are defined. This method is not only logical, but also accurate without misjudgment.

\section{Principle of the computer-aided linkage synthesis}

The working principle of the program package based on Mat$l a b$ is shown in Fig. 1. The first step of linkage synthesis is to input design tasks, i.e. four guiding orientations on sphere or plane. The second step of linkage synthesis is to generate the center points and circle points. Based on the four given orientations, a spherical or planar 4R linkage consisting of poles and image poles is obtained. According to Burmester theory, the center points and the circle points can be obtained by kinematics analysis of the $4 \mathrm{R}$ linkage. The third step of linkage synthesis is to calculate the attributes of all candidate solutions. Number the center points from 1 to $N$ in the order in which it is generated. For any center point $i(i \leq N)$, a candidate linkage solution can be formed by combining any point of the other $(N-1)$ center points. On the basis of it, $N \times(N-1)$ groups of linkage solutions can be formed. For each linkage solution formed by the center point $i$ and the circle point $j(j \leq N, i \neq j)$, the linkage type, the motion defect type and the transmission performance are calculated in turn, and the calculation results are written into the data structure of the linkage solution. After above calculation, the set of candidate solutions is built. The last step of linkage synthesis is to select and analyze linkage solutions. All the candidate linkage solutions are mapped to the solutions map. Any point on the solutions map represents a linkage solution. Its color represents the linkage type and its height represents the level of transmission performance. In the solutions map, all defect solutions are hidden, which improves the efficiency of linkage synthesis. When the user uses the mouse to select a point on the solutions map, the program automatically calculates and displays the corresponding 4R linkage and its coupler curve. Users can watch the animation demonstration of the 4R linkage, or make further analysis of it.

Specially, the synthesis on spherical 4R linkage and planar $4 \mathrm{R}$ linkage are both supported by this program package. Considered the calculation on spherical $4 \mathrm{R}$ linkage is more complex than that on planar 4R linkage, the principle of the synthesis calculation on spherical problem is mainly presented below, and the planar problem is introduced as an extension of the program package.

\section{Generation of the center and circle points}

Based on burmester theory for spherical problems, the circle points are calculated by kinematic analysis of $4 \mathrm{R}$ linkage constructed by image poles. The theory is explained by Shirazi (2005), as shown in Fig. 2. When the driving link of 4R linkage constructed by poles (which is denoted by $\mathrm{P}_{i j}$ ) and image poles (which is denoted by $\mathrm{P}_{i j}^{1}$ ) rotates the angle $\omega$, the 4R linkage moves from the orientation $\mathrm{P}_{12} \mathrm{P}_{13} \mathrm{P}_{34}^{1} \mathrm{P}_{24}^{1}$ to $\mathrm{P}_{12} \mathrm{P}_{13} \mathrm{P}_{34}^{1^{\prime}} \mathrm{P}_{24}^{1^{\prime}}$. The circle point $\mathrm{C}_{1}$ is determined by the intersection of sphere and two planes $\mathrm{P}_{M}$ and $\mathrm{P}_{N}$, the plane $\mathrm{P}_{M}$ is defined by the center point of sphere (which is denoted by point $\mathrm{O}$ ) and normal vector $\mathrm{P}_{24}^{1} \mathrm{P}_{24}^{1^{\prime}}$, the point $\mathrm{P}_{N}$ is defined by the center point of sphere and normal vector $\mathrm{P}_{34}^{1} \mathrm{P}_{34}^{1^{\prime}}$.

For each circle point $\mathrm{C}_{1}$, the center point $\mathrm{C}_{0}$ is obtained as following steps. Firstly, define a point $\mathrm{P}_{123}$ as the reflection point of $\mathrm{C}_{1}$ relative to the plane $\mathrm{P}_{\mathrm{OP}_{12} \mathrm{P}_{13}}$. The plane $\mathrm{P}_{\mathrm{OP}_{12} \mathrm{P}_{13}}$ is defined by the center point of sphere, the pole $\mathrm{P}_{12}$ and $\mathrm{P}_{13}$. And define the point $\mathrm{P}_{124}$ as the reflection point of $\mathrm{C}_{1}$ relative to the plane $\mathrm{P}_{\mathrm{OP}_{12} \mathrm{P}_{14}}$, the plane $\mathrm{P}_{\mathrm{OP}_{12} \mathrm{P}_{14}}$ is defined by the center point of sphere, the pole $\mathrm{P}_{12}$ and $\mathrm{P}_{14}$. Relative to the plane $\mathrm{P}_{\mathrm{OP}_{12} \mathrm{P}_{23}}$, the circle point at the second specified orientation $\mathrm{C}_{2}$ is the reflection point of $\mathrm{P}_{123} \cdot \mathrm{P}_{\mathrm{OP}_{12} \mathrm{P}_{23}}$ is defined by the center point of sphere, the pole $\mathrm{P}_{12}$ and $\mathrm{P}_{23}$. Relative to the plane $\mathrm{P}_{\mathrm{OP}_{13} \mathrm{P}_{23}}$, the circle point at the third specified orientation $\mathrm{C}_{3}$ is the reflection point of $\mathrm{P}_{123} . \mathrm{P}_{\mathrm{OP}_{13} \mathrm{P}_{23}}$ is defined by the center point of sphere, the pole $\mathrm{P}_{13}$ and $\mathrm{P}_{23}$. Relative to the plane $\mathrm{P}_{\mathrm{OP}_{14} \mathrm{P}_{24}}$, the circle point at the fourth specified orientation $\mathrm{C}_{4}$ is the reflection point of $\mathrm{P}_{124}$. $\mathrm{P}_{\mathrm{OP}_{14} \mathrm{P}_{24}}$ is defined by the center point of sphere, the pole $\mathrm{P}_{14}$ and $\mathrm{P}_{24}$. Then, the center point $\mathrm{C}_{0}$ is determined by the intersection of the sphere and any two perpendicular bisector planes of $\mathrm{C}_{1} \mathrm{C}_{2}, \mathrm{C}_{1} \mathrm{C}_{3}$ and $\mathrm{C}_{1} \mathrm{C}_{4}$. With the rotation of the driving link of $4 \mathrm{R}$ linkage $\mathrm{P}_{12} \mathrm{P}_{13} \mathrm{P}_{34}^{1} \mathrm{P}_{24}^{1}$, a series of circle points or center points are generated. Note that two groups of center 


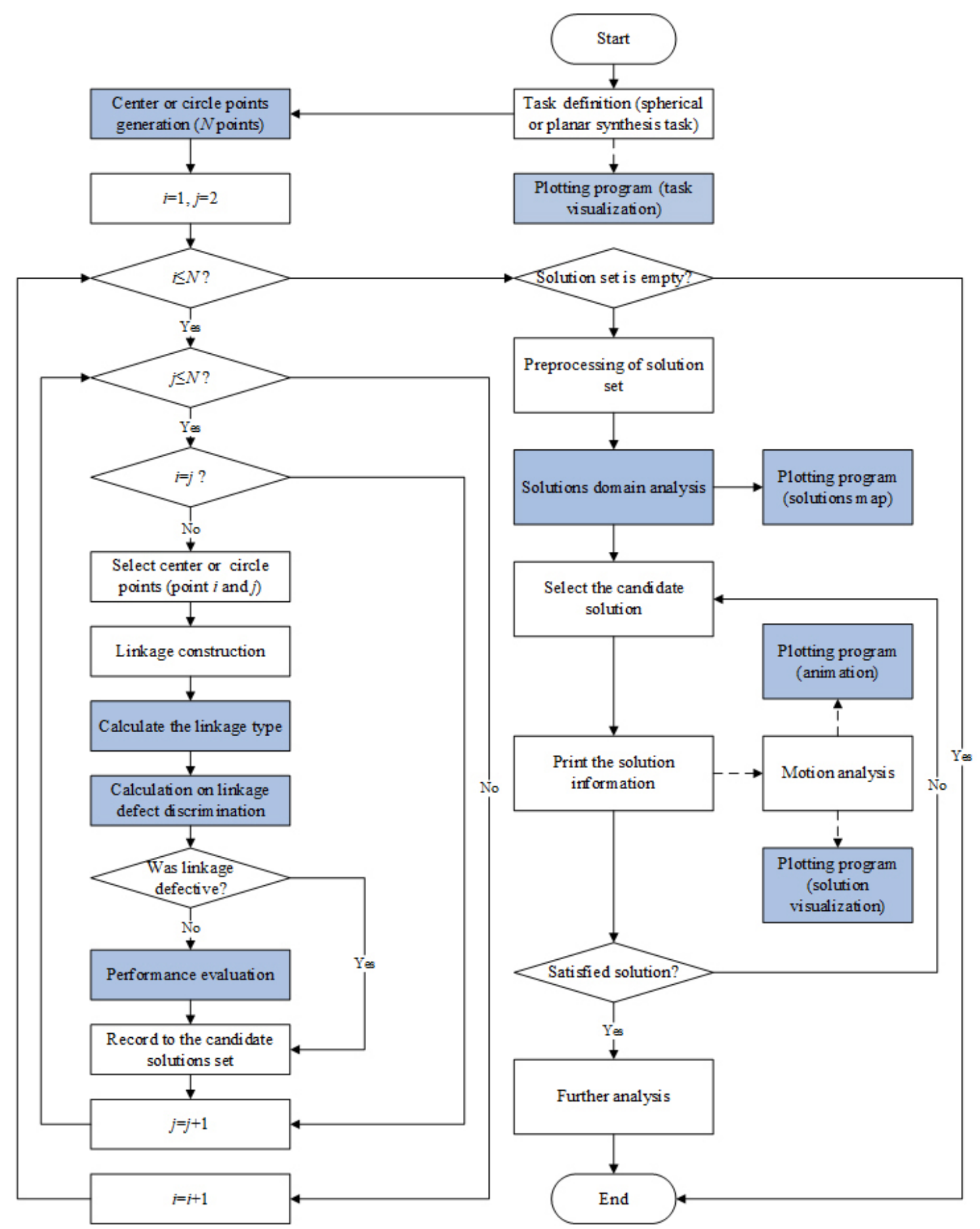

Figure 1. The flow chart of the linkage synthesis calculation.

points and circle points are obtained for each new orientation of linkage $\mathrm{P}_{12} \mathrm{P}_{13} \mathrm{P}_{34}^{1} \mathrm{P}_{24}^{1}$, and they are both valid and symmetrical for the center point of sphere.

In order to realize the calculation and visualization of linkage synthesis mentioned above, a series of functions on geometric calculation are defined in this program package, such as the calculation functions on rotation, mirror, intersection.
4 Calculation and analysis of the linkage solution

\subsection{Circuit defect discrimination}

If the driving link of spherical $4 \mathrm{R}$ linkage rotates partially, there will be a circuit defect when driving link of given four coupler orientations located at different moving intervals. In order to discriminate this motion defect, the limited angles 


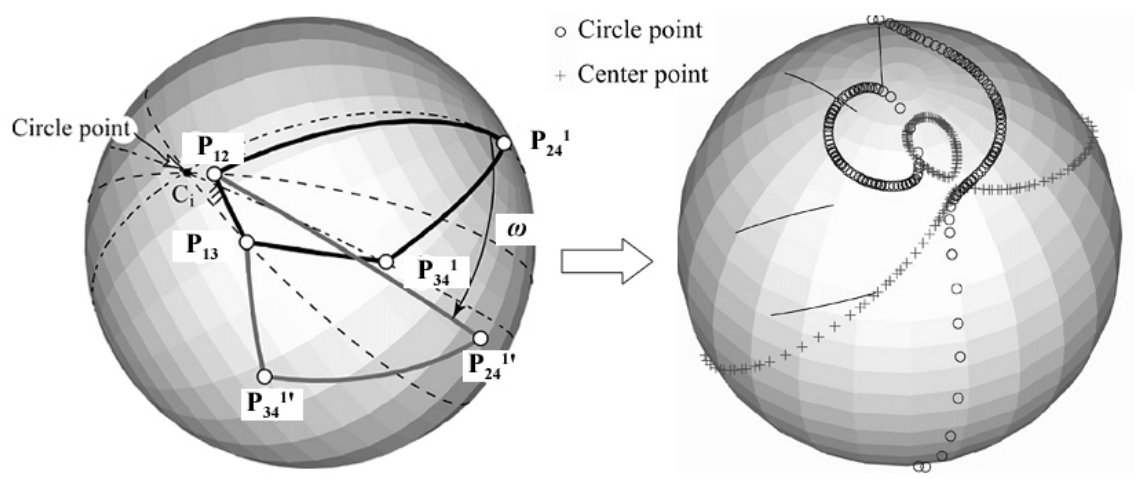

Figure 2. Principle of the spherical 4R linkage synthesis (reproduced from Shirazi, 2007).

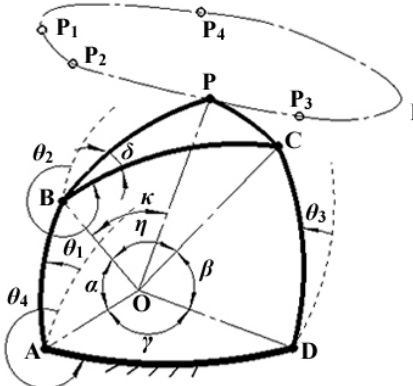

(a) Spherical 4R linkage

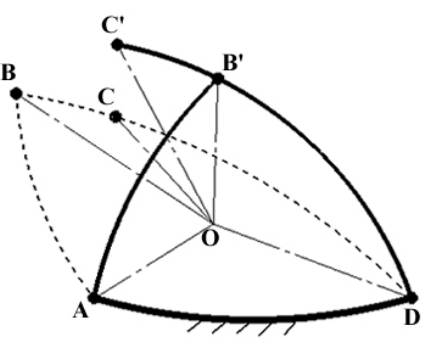

(b) Limited orientations of the driving link
Figure 3. Spherical 4R linkage located at the limited orientations.

of driving link should be calculated firstly. Figure 3 shows a configuration of spherical triangle which is changed from spherical $4 R$ linkage $A B C D(P)$, where $P$ is the guiding point on the coupler link $\mathrm{BC} ; \mathrm{P}_{1}-\mathrm{P}_{4}$ are the given four guiding orientations; $\alpha-\gamma$ and $\theta_{1} \sim \theta_{4}$ represent the length and roll angle of each link of spherical linkage respectively; $\delta$ represents the angle between the link BC and BP. The point coordinates of sphere are defined by longitude $\theta$ and latitude $\varphi$, for example, the coordinates of the point $\mathrm{A}$ can be expressed as $\left(\theta_{\mathrm{A}}\right.$, $\left.\varphi_{\mathrm{A}}\right)$.

For the configuration of spherical triangle $\mathrm{A}(\mathrm{BC}) \mathrm{D}$, define $a=\eta+\beta, b=\gamma, c=\alpha$ and $p=(a+b+c) / 2$, the limited angles of driving link with $\theta_{4}=0$ is calculated by the halfangle formula

$$
\left\{\begin{array}{l}
\sin \frac{\angle A}{2}=\sqrt{\frac{\sin (p-b) \sin (p-c)}{\sin b \sin c}} \\
\cos \frac{\angle A}{2}=\sqrt{\frac{\sin p \sin (p-a)}{\sin b \sin c}}
\end{array}\right.
$$

where $\angle A$ represents the angle from the link $\mathrm{AD}$ to the link AB. In addition, the angle $\theta_{4}$ can be expressed as

$$
\left\{\begin{aligned}
\sin \theta_{4} & =\frac{\cos \phi_{\mathrm{D}} \sin \left(\theta_{\mathrm{A}}-\theta_{\mathrm{D}}\right)}{\sin \gamma} \\
\cos \theta_{4} & =\frac{\sin \phi_{\mathrm{D}}-\sin \phi_{\mathrm{A}} \cos \gamma}{\cos \phi_{\mathrm{A}} \sin \gamma}
\end{aligned}\right.
$$

Then, the limited angles of driving link which is denoted by $\theta_{1}^{+}$and $\theta_{1}^{-}$can be calculated as

$$
\left\{\begin{aligned}
\theta_{1}^{+} & =2 \arctan 2\left(\sin \frac{\angle A}{2}, \cos \frac{\angle A}{2}\right) \\
& +\arctan 2\left(\sin \theta_{4}, \cos \theta_{4}\right) \\
\theta_{1}^{-} & =-2 \arctan 2\left(\sin \frac{\angle A}{2}, \cos \frac{\angle A}{2}\right) \\
& +\arctan 2\left(\sin \theta_{4}, \cos \theta_{4}\right)
\end{aligned}\right.
$$

If there is no real root in Eq. (1), the calculation will be invalid, and the set of the limited angles of the driving link (which is denoted by $\theta_{11}^{*}$ ) will be empty, that is $\theta_{11}^{*}=$ []. If not, $\theta_{11}^{*}=\left[\theta_{1}^{+}, \theta_{1}^{-}\right]$.

Similarly, for the configuration of spherical triangle $\mathrm{A}\left(\mathrm{B}^{\prime} \mathrm{C}^{\prime}\right) \mathrm{D}$, define $a=|\eta-\beta|, b=\gamma, c=\alpha$ and $p=(a+b+$ c) $/ 2$. If there is no real root in Eq. (1), the calculation will be invalid, and the set of the limited angles of the driving link (which is denoted by $\theta_{12}^{*}$ ) will be empty, that is $\theta_{12}^{*}=$ []. If not, $\theta_{12}^{*}=\left[\theta_{1}^{+}, \theta_{1}^{-}\right]$.

The final set of the limited angles of driving link denoted by $\theta_{1}^{*}$, consider $\theta_{1}^{*}=\theta_{11}^{*} \cup \theta_{12}^{*}$. Obviously, the size of $\theta_{1}^{*}$ which is denoted by $N_{1}^{*}$ is only 0,2 or 4 . Due to the driving link can't move between two adjacent moving intervals, the circuit defect can only be checked when $N_{1}^{*}=4$. In this case, if all input angles of the driving link are corresponding to the specified coupler orientations located at the same moving interval, the linkage solution will be valid. If not, there will be a circuit defect in the motion of spherical $4 \mathrm{R}$ linkage. Specially, the input angle of driving link is expressed as

$$
\left\{\begin{array}{l}
\sin \theta_{1}=\frac{\cos \phi_{\mathrm{B}} \sin \left(\theta_{\mathrm{A}}-\theta_{\mathrm{B}}\right)}{\sin \alpha} \\
\cos \theta_{1}=\frac{\sin \phi_{\mathrm{B}}-\sin \phi_{\mathrm{A}} \cos \alpha}{\cos \phi_{\mathrm{A}} \sin \alpha}
\end{array}\right.
$$

\subsection{Branch defect discrimination}

Figure 4 shows two assembly driven link configurations of spherical $4 \mathrm{R}$ linkage $\mathrm{ABCD}$ and $A B C^{\prime} \mathrm{D}$. Due to the linkage is difficult to move from one branch to other without 


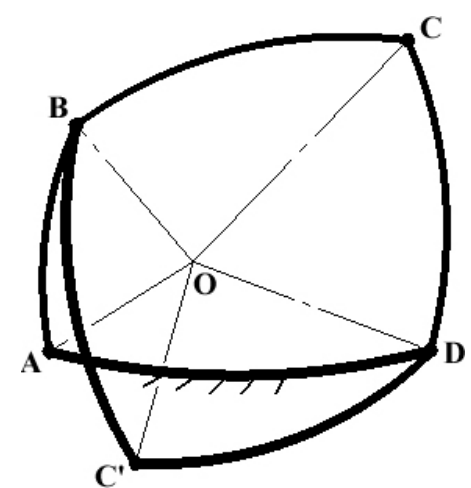

Figure 4. Constructions of the driven link of spherical 4R linkage.

jamming, a branch defect will appear once the driven link in given four coupler orientations locates at different assembly configurations. Actually, the branch of linkage is determined by the solution of motion equation of driven link.

According to the coordinate of point $\mathrm{A}\left(\theta_{\mathrm{A}}, \varphi_{\mathrm{A}}\right)$, the length of $\mathrm{AB}$ (that is $\alpha$ ) and the roll angle of $\mathrm{AB}$ (that is $\theta_{1}$ ), the coordinate of point $\mathrm{B}\left(\theta_{\mathrm{B}}, \varphi_{\mathrm{B}}\right)$ is expressed as

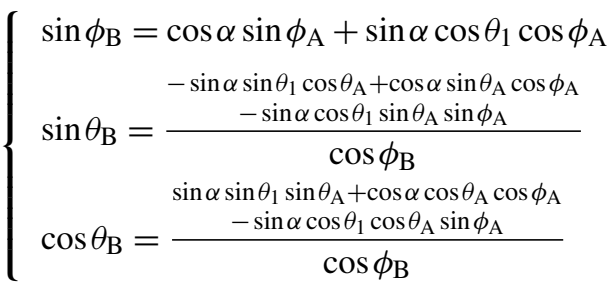

Similarly, the coordinate of point $\mathrm{C}\left(\theta_{\mathrm{C}}, \varphi_{\mathrm{C}}\right)$ can be expressed as

$$
\left\{\begin{aligned}
\sin \phi_{\mathrm{C}} & =\cos \beta \sin \phi_{\mathrm{D}}+\sin \beta \cos \theta_{3} \cos \phi_{\mathrm{D}} \\
\sin \theta_{\mathrm{C}}= & \frac{-\sin \beta \sin \theta_{3} \cos \theta_{\mathrm{D}}+\cos \beta \sin \theta_{\mathrm{D}} \cos \phi_{\mathrm{D}}}{\sin \beta \cos \theta_{3} \sin \theta_{\mathrm{D}} \sin \phi_{\mathrm{D}}} \\
\cos \phi_{\mathrm{C}} & \\
\cos \theta_{\mathrm{C}} & =\frac{-\sin \beta \sin \theta_{3} \sin \theta_{\mathrm{D}}+\cos \beta \cos \theta_{\mathrm{D}} \cos \phi_{\mathrm{D}}}{\cos \phi_{\mathrm{C}}}
\end{aligned}\right.
$$

The relationship between point $\mathrm{B}$ and $\mathrm{C}$ is determined by the length of link BC (that is $\eta$ ), that is

$\cos \eta=\sin \phi_{\mathrm{B}} \sin \phi_{\mathrm{C}}+\cos \phi_{\mathrm{B}} \cos \phi_{\mathrm{C}} \cos \left(\theta_{\mathrm{B}}-\theta_{\mathrm{C}}\right)$

Substitute the Eqs. (5) and (6) into Eq. (7) and obtain following equation, that is

$\tau_{1} \sin \theta_{3}\left(\theta_{1}\right)+\tau_{2} \cos \theta_{3}\left(\theta_{1}\right)+\tau_{3}=0$

Where

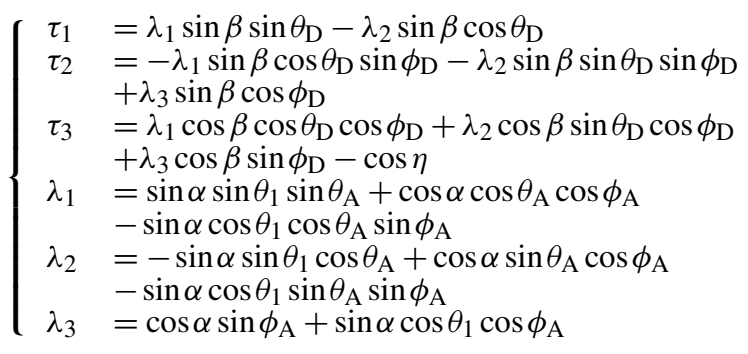

According to above equations, the output angle $\theta_{3}^{+}$obtains two roots that is $\theta_{3}^{+}$and $\theta_{3}^{-}$, which can be calculated as

$$
\left\{\begin{array}{l}
\theta_{3}^{+}\left(\theta_{1}\right)=2 \arctan \left(\frac{\tau_{1}+\sqrt{\tau_{1}^{2}+\tau_{2}^{2}-\tau_{3}^{2}}}{\tau_{2}-\tau_{3}}\right) \\
\theta_{3}^{-}\left(\theta_{1}\right)=2 \arctan \left(\frac{\tau_{1}-\sqrt{\tau_{1}^{2}+\tau_{2}^{2}-\tau_{3}^{2}}}{\tau_{2}-\tau_{3}}\right)
\end{array}\right.
$$

For each location of driving link corresponded to four specified coupler orientations, if all actual output angles $\theta_{3}$ are generated by the same subformula of Eq. (10), the linkage solution will be valid. If not, a branch defect will appear in the motion of spherical $4 \mathrm{R}$ linkage. Specially, according to the coordinates of point $\mathrm{C}$ and $\mathrm{D}$, the actual output angles $\theta_{3}$ can be calculated by the method of Eq. (4).

\subsection{Order defect discrimination}

The order defect occurs when the coupler link can't pass the given four-orientations with the order of $1-2-3-4$ or 4-3$2-1$. Obviously, if the driving link is rotated fully, the geometrical criterion $\theta_{21}<\theta_{31}<\theta_{41}$ or $\theta_{21}>\theta_{31}>\theta_{41}$ will be used to solve this problem according to the definition of order defect, where $\theta_{j i}$ represents the rotation angle of driving link revolves from orientation $\mathrm{AB}^{i}$ (which is corresponded to coupler orientation $i$ ) to $\mathrm{AB}^{j}$ (which is corresponded to coupler orientation $j$ ) round the axis OA, as shown in Fig. 5.

In order to obtain rotation angle $\theta_{j i}$, the normal vector of plane $\mathrm{OAB}^{i}$ (which is denoted by $n_{1}$ ) and $\mathrm{OAB}^{j}$ (which is denoted by $n_{2}$ ) are expressed as $n_{1}=\mathrm{OA} \times \mathrm{OB}^{i}, n_{2}=\mathrm{OA} \times$ $\mathrm{OB}^{j}$. Based on the conclusions, the normal vector of axis $\mathrm{OA}$ in plane $\mathrm{OAB}^{i}$ (which is denoted by $f_{1}$ ) and plane $\mathrm{OAB}^{j}$ (which is denoted by $f_{2}$ ) are expressed as $f_{1}=n_{1} \times \mathrm{OA}, f_{2}=$ $n_{2} \times$ OA. Define OA $=\left[x_{1}, y_{1}, z_{1}\right]$, and $f_{1} \times f_{2}=\left[x_{2}, y_{2}, z_{2}\right]$. The rotation angle of driving link $\theta_{j i}$ is calculated as

$$
\left\{\begin{array}{cc}
\theta_{j i}=\arccos \frac{f_{1} \cdot f_{2}}{\left|f_{1}\right|\left|f_{2}\right|} & z_{1} \cdot z_{2}>0 \\
\theta_{j i}=2 \pi-\theta_{j i} & \text { others }
\end{array}\right.
$$

On the contrary, if the driving link is rotated partially, the geometrical criterion which mentioned above (that is $\theta_{21}<$ $\theta_{31}<\theta_{41}$ or $\left.\theta_{21}>\theta_{31}>\theta_{41}\right)$ will be invalid. For example, 


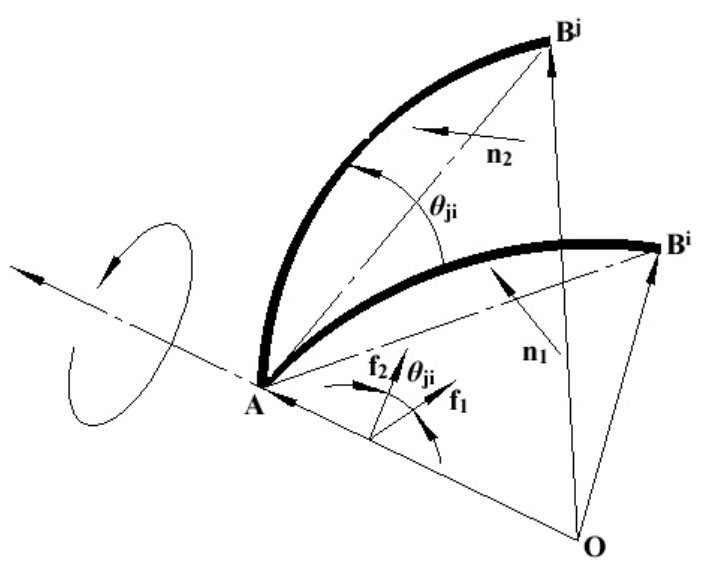

Figure 5. Driving link revolved round the pole axis of sphere.

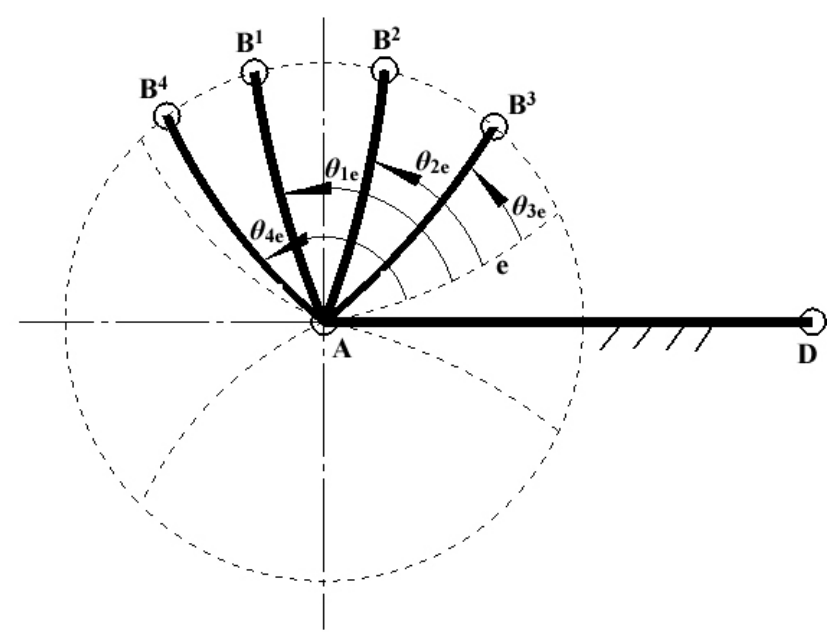

Figure 6. Order defect discrimination.

the order 4-1-2-3 is incorrect because of the driving link can't move from one moving interval to other, as shown in Fig. 6. In this case, the geometrical criterion is modified as $\theta_{1 e}<\theta_{2 e}<\theta_{3 e}<\theta_{4 e}$ or $\theta_{1 e}>\theta_{2 e}>\theta_{3 e}>\theta_{4 e}$, where the subscript letter $e$ represents the arbitrary limited orientations of the driving link.

\subsection{Pyramid structure of the motion defect calculation}

The method of defect discrimination cannot be considered in isolation. For example, the calculation on the order defect discrimination is meaningless for a $4 \mathrm{R}$ linkage with circle defect because the coupler of the $4 \mathrm{R}$ linkage cannot pass through all specified orientations. In order to solve this problem, a pyramid structure is defined in this program package. The circuit defect locates at the bottom of pyramid, the branch defect locates at the middle of pyramid, and the order defect locates at the top of pyramid. Under this definition, both the circuit defect and branch defect should be checked first before the calculation of order defect discrim-
Table 1. Classification of the linkage type (Murray and Larochelle, 1998).

\begin{tabular}{lcccc}
\hline & $K_{1}$ & $K_{2}$ & $K_{3}$ & $K_{4}$ \\
\hline Crank rocker & $+(-)$ & $+(-)$ & $+(-)$ & $+(-)$ \\
Rocker crank & $+(-)$ & $-(+)$ & $-(+)$ & $+(-)$ \\
Double crank & $-(+)$ & $-(+)$ & $+(-)$ & $+(-)$ \\
Grashof double rocker & $-(+)$ & $+(-)$ & $-(+)$ & $+(-)$ \\
00 double rocker & $-(+)$ & $-(+)$ & $-(+)$ & $+(-)$ \\
$0 \pi$ double rocker & $+(-)$ & $+(-)$ & $-(+)$ & $+(-)$ \\
$\pi 0$ double rocker & $+(-)$ & $-(+)$ & $+(-)$ & $+(-)$ \\
$\pi \pi$ double rocker & $-(+)$ & $+(-)$ & $+(-)$ & $+(-)$ \\
\hline
\end{tabular}

ination. Similarly, the circuit defect should be checked first before the calculation of branch defect discrimination.

\subsection{Linkage type classification}

For a number of synthesis tasks, the $4 \mathrm{R}$ linkage with specified linkage type should be satisfied. According to the method proposed by Murray, there are 8 kinds of linkage type for a spherical $4 \mathrm{R}$ linkage, as shown in Table 1 , where $K_{1}=\gamma-\alpha+\eta-\beta, K_{2}=\gamma-\alpha-\eta+\beta, K_{3}=\eta+\beta-\gamma-\alpha$, $K_{4}=2 \pi-\eta-\beta-\gamma-\alpha$. Specially, at least one link of a $4 \mathrm{R}$ linkage will be rotated fully if $K_{1} K_{2} K_{3} K_{4}>0$, which is the Grashof's condition. Both calculations on the linkage type and Grashof's condition are supported by program package.

\subsection{Performance evaluation and solutions domain analysis}

A standard function interface on linkage performance calculation is provided for the designer in the program package. The input vector of this function is a data structure to describe the coordinate information of linkage solution at each specified orientation, and the output variable of this function is a real number to indicate the linkage performance. If there are more than one evaluating indicators for the linkage performance, a weight method will be used. Of course, all the functions in the program package can be called in this function.

Actually, a default function template is provided for the designer. The evaluating indicator which defined in this function template is termed as "Braking angle". For a valid 4R linkage solution which driving link is rotated partially, if the rotation angle of driving link from the specified orientation 1 (or 4) to the nearest limited orientation is too small, the driving link will be difficult to stop before the linkage moves to the limited orientation for the reason of the high rotation speed or the inaccurate displacement control of the motor.

If $N$ center points and circle points are generated after the synthesis calculation, $N \times N$ linkage solutions in total will be obtained. Based on the calculation on linkage performance evaluation, each linkage solution will be analyzed and the 


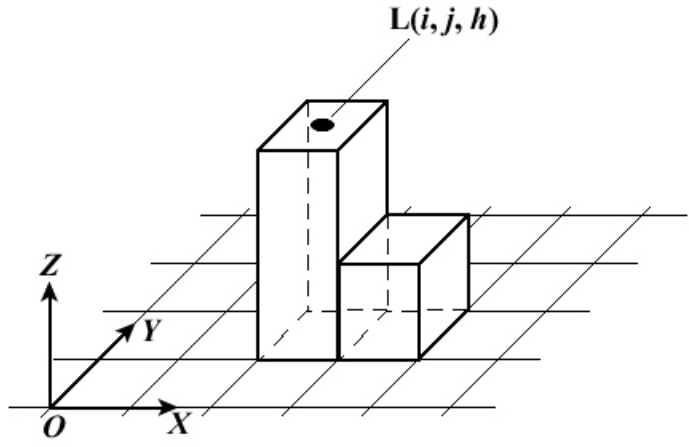

Figure 7. Constructions of solutions map.

distribution of the linkage performance evaluation in the solution domain will be obtained. The calculation on the solutions domain analysis is visualized by a solutions map, which is introduced later.

\section{Synthesis visualization}

\subsection{Task visualization}

Once the four coupler orientations is specified, a figure with four orientations, the center points and the circle points plotted in a sphere are generated. The designer can adjust task parameters according to the figure. The specified coupler orientations are defined by four equilong arcs in the sphere, and the longitude and latitude of the coupler orientation are determined by the starting point of arc, and the roll angle of coupler orientation is determined by the roll angle of arc. Specially, in order to plot the arc in the surface of sphere smoothly, the arc is divided into several line segments through an interpolation method.

\subsection{Solutions map}

For $N$ center points and circle points, all linkage solutions can be mapped into a three-dimensional coordinate system $O-X Y Z$, that is the visualization of solutions domain analysis mentioned above. In this coordinate system, $N \times N$ cubes are constructed based on the $O-X Y$ plane, and each linkage solution defined by the center point (or circle point) $i$ and the center point (or circle point) $j$ is described by a cube $L(i, j, h)$, as shown in Fig. 7, where $i$ is the $x$ offset of cube $L, j$ is the $y$ offset of the cube $L, h$ is the $z$ offset of the cube $L, i$ and $j$ are determined by selected center points (or circle points), and $h$ is determined by linkage performance. In order to describe the linkage type of solution, each cube in the solutions map is color-coded according to linkage type.

\subsection{Solutions visualization}

The key problem of solutions visualization is the motion analysis and calculation of coupler curve. Figure 3 shows that the output angle $\theta_{3}$ is calculated by the Eq. (10) aimmed at each input angle $\theta_{1}$. Due to the coordinates of point $\mathrm{A}$ and $\mathrm{D}$ of spherical $4 \mathrm{R}$ linkage are given, the coordinates of point $\mathrm{B}$ and $\mathrm{C}$ can be obtained according to the Eqs. (5) and (6). Then, the rotation angle of link BC (that is $\theta_{2}$ ) can be calculated as

$$
\left\{\begin{aligned}
\sin \theta_{2} & =\frac{\cos \phi_{\mathrm{C}} \sin \left(\theta_{\mathrm{B}}-\theta_{\mathrm{C}}\right)}{\sin \eta} \\
\cos \theta_{2} & =\frac{\sin \phi_{\mathrm{C}}-\sin \phi_{\mathrm{B}} \cos \eta}{\cos \phi_{\mathrm{B}} \sin \eta}
\end{aligned}\right.
$$

Correspondingly, the rotation angle revolved from the link $\mathrm{BC}$ to $\mathrm{BP}$ (that is $\delta$ ) is obtained by the rotation method introduced in Sect. 3.3. Obviously, the angle $\delta$ is a constant value and calculated from the coordinate information of spherical 4R linkage at any specified orientations. On this basis, the rotation angle of link BP (denoted by $\theta_{5}$ ) is " $\theta_{2}+\delta$ ", and the coordinate of point $\mathrm{P}$ is expressed as

$$
\left\{\begin{aligned}
& \sin \phi_{\mathrm{P}}= \cos \kappa \sin \phi_{\mathrm{B}}+\sin \kappa \cos \theta_{5} \cos \phi_{\mathrm{B}} \\
& \sin \theta_{\mathrm{P}}= \frac{-\sin \kappa \sin \theta_{5} \cos \theta_{\mathrm{B}}+\cos \kappa \sin \theta_{\mathrm{B}} \cos \phi_{\mathrm{B}}}{\sin \kappa \cos \theta_{5} \sin \theta_{\mathrm{B}} \sin \phi_{\mathrm{B}}} \\
& \cos \phi_{\mathrm{P}} \\
& \cos \theta_{\mathrm{P}}= \frac{-\sin \kappa \sin \theta_{5} \sin \theta_{\mathrm{B}}+\cos \kappa \cos \theta_{\mathrm{B}} \cos \phi_{\mathrm{B}}}{\cos \phi_{\mathrm{P}}}
\end{aligned}\right.
$$

According to the calculation above, the point coordinates of the spherical 4R linkage under each input angle are calculated, and the coordinate data is applied to conduct the animation of linkage motion and plot the coupler curve. Due to there are two solutions in the Eq. (10) corresponding to the two assembly configurations of spherical $4 \mathrm{R}$ linkage driven link, the coupler curve is generated at twice. In order to distinguish the branch of coupler curve, different colors are used to represent different branches. Specially, for a spherical $4 \mathrm{R}$ linkage which driving link rotated partially, each solution of Eq. (10) should be checked because there is no real root for some input angles of driving link.

\section{Expansion of the program package on planar 4R linkage}

The synthesis on both spherical and planar 4R linkages is supported by the program package. The calculation on spherical problem mentioned above is mainly based on the theory of spherical trigonometry (Ratcliffe, 2006). Actually, the geometrical principle of planar 4R linkage is similar to a spherical one. As the Fig. 2 shows, the spherical 4R linkage only move along the surface of a sphere and all the axes of hinges passes through the center of sphere. If the diameter of sphere is infinite, the spherical $4 \mathrm{R}$ linkage $\mathrm{ABCD}$ will be transformed into a planar one.

For the synthesis of planar 4R linkage, the method mentioned in Sect. 2 which is presented by Shirazi (2005) is applicable, it is based on the classic burmester theory and cannot be described here. 
For the discrimination of linkage circuit defect, the difference between spherical and planar problems is the calculation of limited orientations of driving link, which can be obtained by the cosine theorem of planar triangle. Define $a^{\prime}=|\mathrm{BC}|+|\mathrm{CD}|, b^{\prime}=|\mathrm{AD}|, c^{\prime}=|\mathrm{AB}|$, if the configuration of planar triangle is $\mathrm{A}(\mathrm{BC}) \mathrm{D}$, the limited angles of driving link which is denoted by $\theta_{1}^{1 *}$ and $\theta_{1}^{2 *}$ could be calculated as

$$
\left\{\begin{array}{l}
\theta_{1}^{1 *}=\arccos \left(\frac{c^{\prime 2}+b^{\prime 2}-a^{\prime 2}}{2 c^{\prime} b^{\prime}}\right)+\arctan 2\left(\sin \theta_{4}, \cos \theta_{4}\right) \\
\theta_{1}^{2 *}=-\arccos \left(\frac{c^{\prime 2}+b^{\prime 2}-a^{\prime 2}}{2 c^{\prime} b^{\prime}}\right)+\arctan 2\left(\sin \theta_{4}, \cos \theta_{4}\right)
\end{array}\right.
$$

Similarly, if the configuration of planar triangle is $\mathrm{A}\left(\mathrm{B}^{\prime} \mathrm{C}^{\prime}\right) \mathrm{D}$, the Eq. (14) will be applicable as $a=|\mathrm{BC}|-|\mathrm{CD}|$. If it is not all input angles of driving link corresponding to the specified coupler orientations locates at the same moving interval, the linkage solution will be invalid. Specially, the validity of Eq. (14) is determined by the validity of expression $v=\left(c^{2}+b^{2}-a^{2}\right) / 2 c b$. If the solution of Eq. (14) is valid, the condition $v \in[-1,1]$ should be satisfied.

For the discrimination of linkage branch defect, the Eqs. (8) and (10) are applicable, the difference between spherical and planar problems is the expression of $\tau_{1}, \tau_{2}, \tau_{3}$, where

$$
\left\{\begin{aligned}
\tau_{1} & =2|\mathrm{AB}||\mathrm{CD}| \sin \theta_{1}-2|\mathrm{AD}||\mathrm{CD}| \sin \theta_{4} \\
\tau_{2} & =2|\mathrm{AB}||\mathrm{CD}| \cos \theta_{1}-2|\mathrm{AD}||\mathrm{CD}| \cos \theta_{4} \\
\tau_{3} & =|\mathrm{BC}|^{2}-|\mathrm{AB}|^{2}-|\mathrm{CD}|^{2}-|\mathrm{AD}|^{2} \\
& +2|\mathrm{AB}||\mathrm{AD}| \cos \left(\theta_{1}-\theta_{4}\right)
\end{aligned}\right.
$$

If it was not all actual output angles $\theta_{3}$ corresponding to four specified coupler orientations that could be generated by the same subformula of Eq. (14), the linkage solution would be invalid.

For the discrimination of linkage order defect, the geometrical criterion $\theta_{21}<\theta_{31}<\theta_{41}$ or $\theta_{21}>\theta_{31}>\theta_{41}$ for linkage with rotating fully driving link and geometrical criterion $\theta_{1 e}<\theta_{2 e}<\theta_{3 e}<\theta_{4 e}$ or $\theta_{1 e}>\theta_{2 e}>\theta_{3 e}>\theta_{4 e}$ for linkage with rotating partially driving link are applicable. The difference between spherical and planar problems is the vectors $f_{1}$ and $f_{2}$ in the Eq. (11), where $f_{1}=\mathrm{AB}^{i}$ and $f_{2}=$ $\mathrm{AB}^{j}$.

For the classification of linkage type and the condition of grashof, the method proposed by Martin and Murray (2002) is applicable to planar $4 \mathrm{R}$ linkage. For the performance evaluation, the "Braking angle" of planar 4R linkage is obtained from the calculation on planar vectors. Similarly, for the synthesis visualization of planar $4 \mathrm{R}$ linkage, the method mentioned in Sect. 4 is applicable, and the calculation is based on the principle of planar trigonometry.

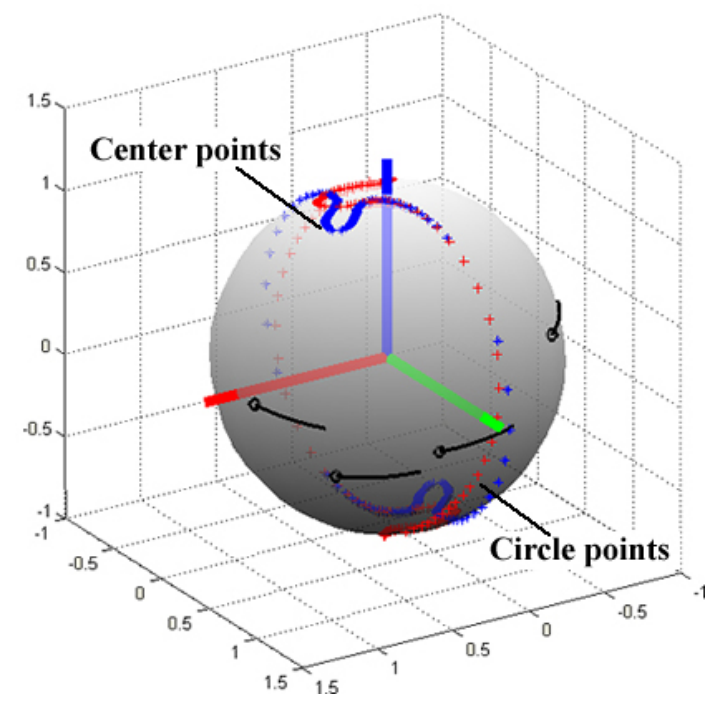

Figure 8. Center points and circle points generated by the program package.

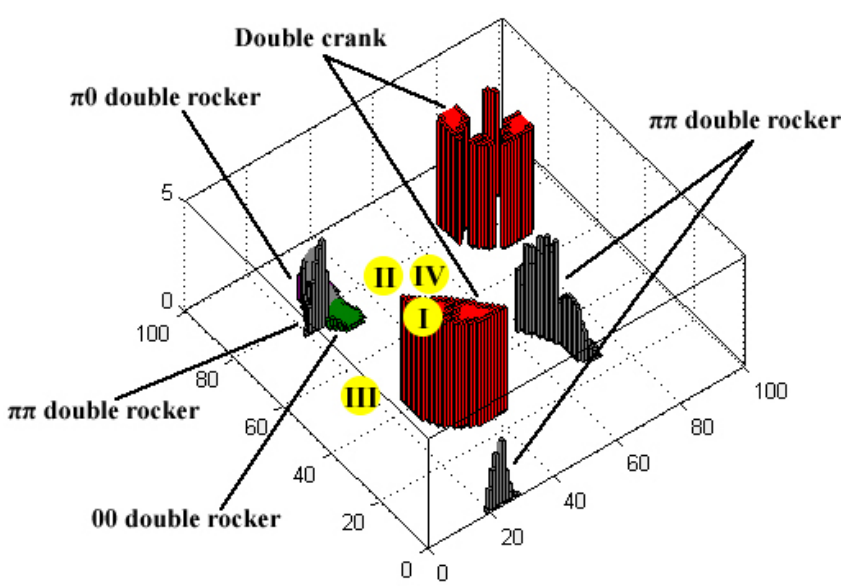

Figure 9. Solutions map generated by the program package.

\section{Examples}

\subsection{Spherical 4R linkage synthesis}

In this example, it is necessary to synthesize a spherical $4 \mathrm{R}$ linkage to guide a camera to pass four specified orientations without circuit defect, branch defect and order defect, as shown in Table 2.

Firstly, the synthesis program is called to generate center points and circle points, the result is shown in Fig. 8. Then, the solutions map is generated, the result is shown in Fig. 9. As shown in Fig. 9, 91.7\% of solutions are erased from the map because of the linkage motion defect, and there are no valid linkages with type of crank-rocker, rocker crank, grashof double rocker and $0 \pi$ double rocker satisfied the synthesis task. Additionally, all linkage solutions which driving link rotate partially have a bad "Braking angle" less than $5^{\circ}$, 


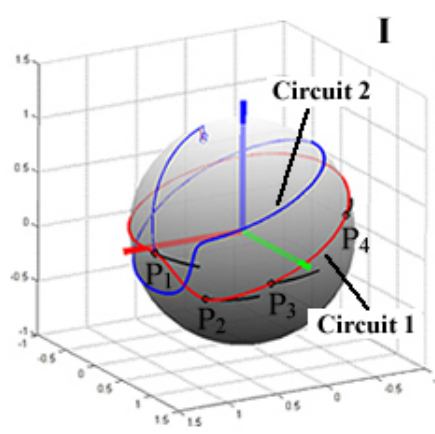

(a) Valid solution

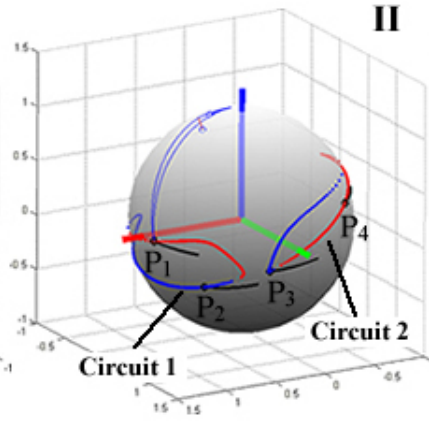

(b) Circuit defect

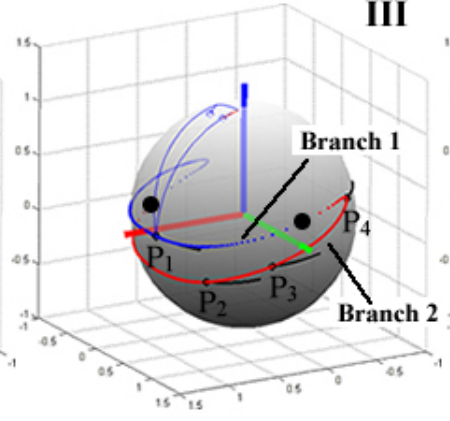

(c) Branch defect

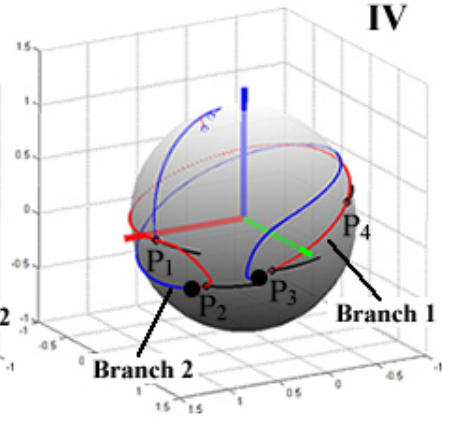

(d) Order defect

Figure 10. Solutions visualization.

Table 2. Specified coupler orientations for a spherical $4 \mathrm{R}$ linkage.

\begin{tabular}{lrrr}
\hline Orientation & Longitude $\left(^{\circ}\right)$ & Latitude $\left(^{\circ}\right)$ & Roll $\left(^{\circ}\right)$ \\
\hline $\mathrm{P}_{1}$ & 10 & 0 & -4 \\
$\mathrm{P}_{2}$ & 40 & -20 & 1 \\
$\mathrm{P}_{3}$ & 75 & -10 & 4 \\
$\mathrm{P}_{4}$ & 130 & 15 & 5 \\
\hline
\end{tabular}

therefore, only the solutions with a type of double crank need to be considered.

The program allows designer to select valid solutions on the solutions map directly, and this way is quick and efficient. As the Fig. 9 shows, a candidate solution is selected from region I of solutions map, and the solution information is shown in Table 3. Similarly, another three linkage solutions selected from region II-IV of solutions map are erased because of motion defect, and the solution information is shown in Table 3. In order to verify the results, the plotting program is called to generate coupler curve of four linkages, as shown in Fig. 10. Obviously, only the linkage solution I is feasible.

In this calculation, 7396 groups of linkage solutions are synthesized and analyzed. When the program is running on the computer, it takes $4.48 \mathrm{~s}$ to generate and display the solutions map at one time. In the process of linkage solution selection, it only takes $0.37 \mathrm{~s}$ to generate and display the coupler curve of the selected linkage solution each time.

\subsection{Planar 4R linkage synthesis}

In this example, it is necessary to synthesize a planar $4 \mathrm{R}$ linkage to guide the bucket of the skid steer loader to pass four specified orientations without circuit defect, branch defect and order defect, as shown in Table 4.

Firstly, the synthesis program is called to generate center points and circle points, the result is shown in Fig. 11. Then, the solutions map is generated, the result is shown in Fig. 12. It is noted that the installation position of the $4 R$ linkage on the skid steer loader is limited, which becomes a strong constraint condition for the rationality of the solution.

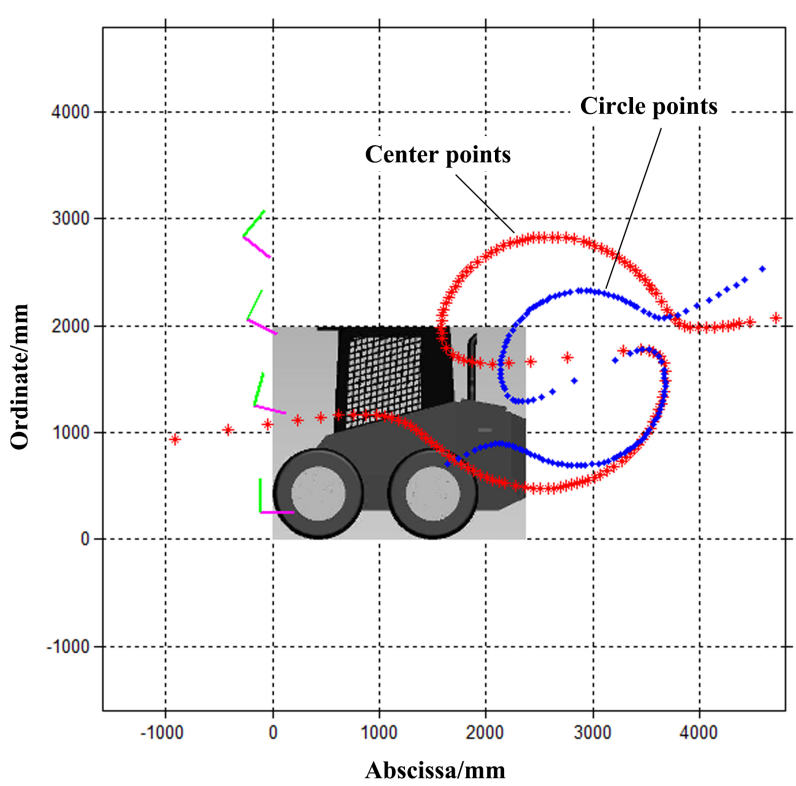

Figure 11. Center points and circle points generated by the program package.

In order to provide enough candidate solutions, this example no longer calculates the transmission performance of the linkage solution, but loads the picture of the skid steer loader with reduced proportion into the drawing area to evaluate the reasonableness of the installation position of the linkage solution.

As shown in Fig. 12, $70.95 \%$ of solutions are erased from the map because of the linkage motion defect, and there are no valid linkages with type of crank-rocker, rocker crank, double crank, 00 double rocker and $\pi \pi$ double rocker satisfied the synthesis task. Six candidate solutions are selected from region I-VI of solutions map, and the solution information is shown in Table 5. In order to verify the results, the plotting program is called to generate coupler curve of six linkages. As shown in Fig. 13, the installation position of one rocker in solution II interferes with the tire of the skid steer loader, and the installation position of one rocker in solution 
Table 3. Solutions information.

\begin{tabular}{lrrrrrl}
\hline No. & $\mathrm{A}\left(\theta_{\mathrm{A}}, \varphi_{\mathrm{A}}\right)\left(^{\circ}\right)$ & $\mathrm{B}\left(\theta_{\mathrm{B}}, \varphi_{\mathrm{B}}\right)\left(^{\circ}\right)$ & $\mathrm{C}\left(\theta_{\mathrm{C}}, \varphi_{\mathrm{C}}\right)\left(^{\circ}\right)$ & $\mathrm{D}\left(\theta_{\mathrm{D}}, \varphi_{\mathrm{D}}\right)\left(^{\circ}\right)$ & Defection type & Linkage type \\
\hline I & $2.7341,65.4782$ & $-39.3638,67.3055$ & $-49.9400,68.4720$ & $-4.1762,67.9088$ & No defect & Double crank \\
II & $6.0954,64.7366$ & $-34.2196,67.5189$ & $-85.6241,80.5367$ & $-31.0304,73.7294$ & Circle defect & Roker crank \\
III & $21.0157,72.8766$ & $35.1467,83.0991$ & $-63.4609,73.7618$ & $-13.2424,71.8844$ & Branch defect & $0 \pi$ double rocker \\
IV & $6.0954,64.7366$ & $-34.2196,67.5189$ & $-68.0395,75.9051$ & $-16.5879,72.9075$ & Order defect & 00 double rocker \\
\hline
\end{tabular}

Table 4. Specified coupler orientations for a planar 4R linkage.

\begin{tabular}{lrrr}
\hline Orientation & Abscissa $(\mathrm{mm})$ & Ordinate $(\mathrm{mm})$ & Roll $\left({ }^{\circ}\right)$ \\
\hline $\mathrm{P}_{1}$ & -112 & 246 & 0 \\
$\mathrm{P}_{2}$ & -179 & 1260 & -15.8 \\
$\mathrm{P}_{3}$ & -239 & 2060 & -26.5 \\
$\mathrm{P}_{4}$ & -277 & 2838 & -39.1 \\
\hline
\end{tabular}

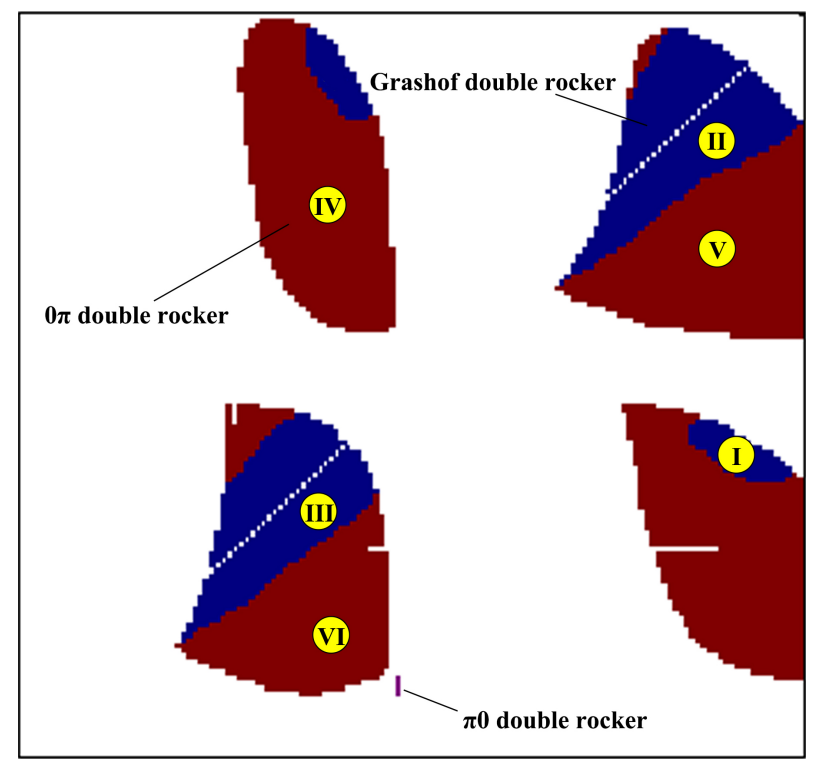

Figure 12. Solutions map generated by the program package.

III-VI is outside the body of the skid steer loader. Obviously, only the installation position of the rocker with solution I is reasonable and meets the requirements of linkage synthesis.

In this calculation, 19600 groups of linkage solutions are synthesized and analyzed. When the program is run on the computer, it takes $12.73 \mathrm{~s}$ to generate and display the solutions map at one time. In the process of linkage solution selection, it only takes $0.24 \mathrm{~s}$ to generate and display the coupler curve of the selected linkage solution each time.

\section{Concluding remarks}

In this paper, a synthesis program package is developed to find a satisfied planar or spherical linkage solution automat- ically according to burmester theory. The main conclusions of this study are as follows:

1. The program package developed in this paper has fast calculation speed and high synthesis efficiency. The time required for synthesis calculation depends on the segmentation accuracy of buermester center curve, that is, the rotation angle increment of driving link of $4 \mathrm{R}$ linkage constructed by image poles when generating each center point. The more precise the burmester center curve is segmented, the more solutions are generated and the longer the calculation time is. In the two examples given in this paper, the number of linkage solutions analyzed is 7396 and 19600 respectively, and the total time required for solutions map generation and display is 4.48 and $12.73 \mathrm{~s}$ respectively. The generation of the solutions map is one-time, after which the selection and analysis of linkage solution only takes less than $0.5 \mathrm{~s}$ each time. In addition, the solutions map proposed in this study extends the dimension of traditional type map, so that it can also express the transmission performance of linkage solutions while expressing linkage types. This method further avoids the blindness of linkage synthesis.

2. The proposed defect discrimination algorithm is not only correct but also logical. In the synthetic example of spherical 4R linkage, by comparing the result of program discrimination with that of coupler curve analysis, it can be seen that the algorithm in this paper can correctly discriminate the defect of circuit, branch and order. Moreover, this paper proposes a pyramid structure for defect discrimination. This is obviously more logical. For example, when four given orientations are located in different circuits and branches, the coupler curve of $4 \mathrm{R}$ linkage can not pass through all four orientations at all. It is not logical to discuss whether the coupler link can pass through four orientations orderly.

3. The program developed in this paper is more flexible than the previous software. Like the toolbox functions of MATLAB, the program contains a series of functions from the bottom graphics calculation to the generation of human-computer interaction interface. On this basis, users can not only directly use the given humancomputer interaction interface for general synthesis cal- 
Table 5. Solutions information.

\begin{tabular}{lrrrrl}
\hline No. & $\mathrm{A}(x, y)(\mathrm{mm})$ & $\mathrm{B}(x, y)(\mathrm{mm})$ & $\mathrm{C}(x, y)(\mathrm{mm})$ & $\mathrm{D}(x, y)(\mathrm{mm})$ & Linkage type \\
\hline I & $1291.0013,1059.097$ & $2278.2537,860.1666$ & $2172.8115,1402.3409$ & $1589.9643,1884.2771$ & Grashof double rocker \\
II & $1341.0647,1026.7513$ & $2310.2136,846.3586$ & $2625.8126,720.3957$ & $1763.8252,707.1583$ & Grashof double rocker \\
III & $1581.3274,1933.6541$ & $2159.8975,1434.7617$ & $2158.3788,1759.3985$ & $1709.5081,2371.6734$ & Grashof double rocker \\
IV & $1581.3274,1933.6541$ & $2159.8975,1434.7617$ & $3356.0852,849.1585$ & $2853.9685,523.0526$ & $0 \pi$ double rocker \\
V & $1341.0647,1026.7513$ & $2310.2136,846.3586$ & $3535.195,1029.9644$ & $3194.5167,681.381$ & $0 \pi$ double rocker \\
VI & $1589.9643,1884.2771$ & $2172.8115,1402.3409$ & $3018.7688,2319.3973$ & $3133.9593,2700.2595$ & $0 \pi$ double rocker \\
\hline
\end{tabular}

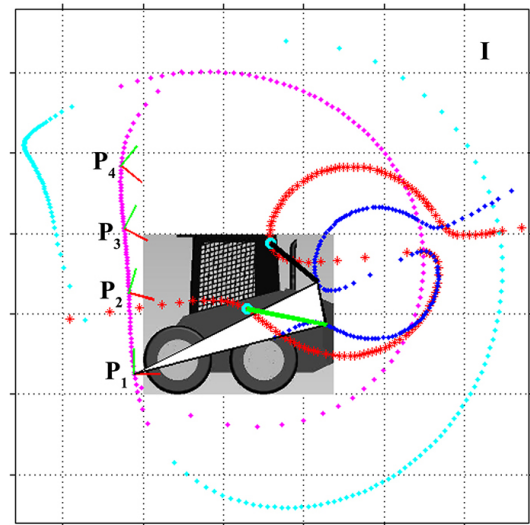

(a) Solution I

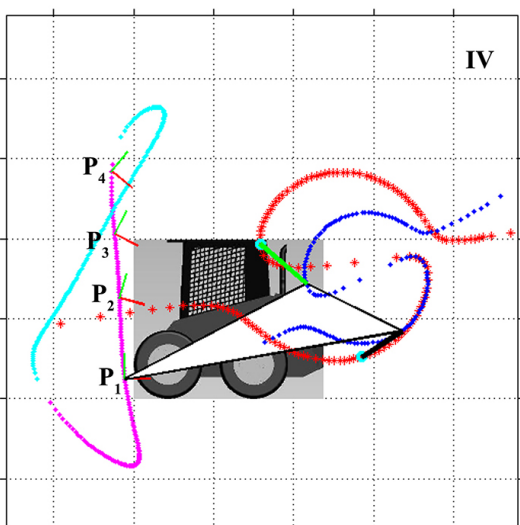

(d) Solution IV

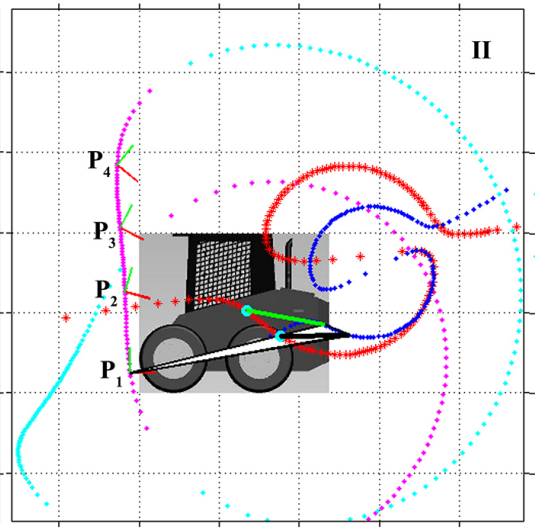

(b) Solution II

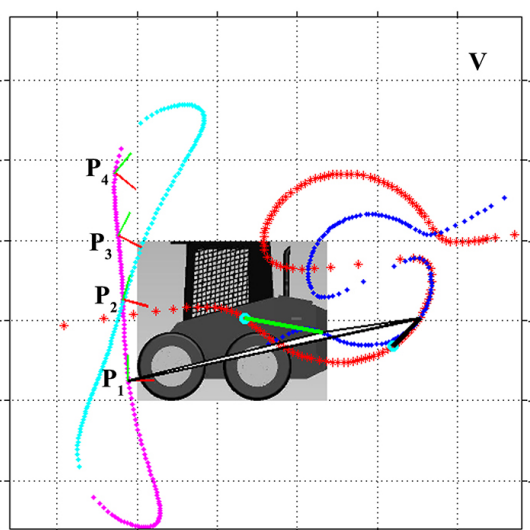

(e) Solution V

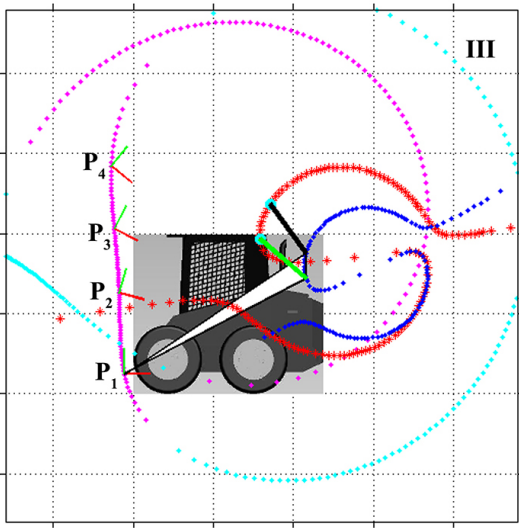

(c) Solution III

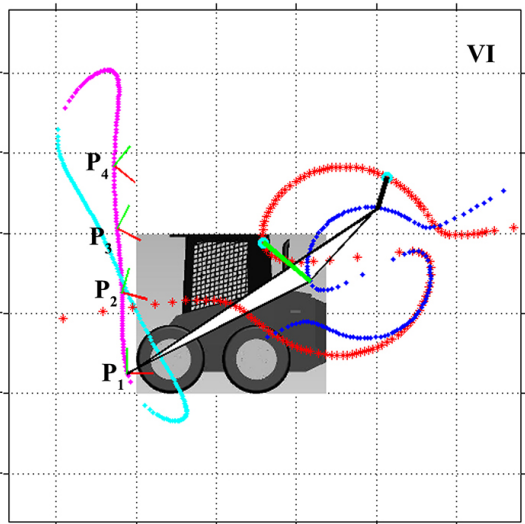

(f) Solution VI

Figure 13. Solutions visualization.

culation, but also reorganize these functions to solve more complex problems.

Considering that the precise orientation synthesis problem and the function synthesis problem can be transformed each other, how to extend the research method in this paper, especially the defect discrimination method, to the function synthesis field of spherical and planar 4R linkage is the next research direction of this paper.

Data availability. All the data used in this paper can be obtained by request from the corresponding author.
Author contributions. GW suggested the overall concept of this paper and completed the development of the program package based on Matlab. HZ, XL and JW worked together to complete the examples used in this paper. $\mathrm{XZ}$ and GF edited and verified all the formulas and pictures used in this paper.

Competing interests. The authors declare that they have no conflict of interest.

Acknowledgements. This work is financially supported by the National Key Research and Development Program (Project no. 2016YFD0701103), the Shandong Key Research and Development 
Program (Project no. 2018GNC112008), and the Funds of Shandong "Double Tops" Program (Project no. SYL2017XTTD14).

Financial support. This research has been supported by the National Key Research and Development Program (grant no. 2016YFD0701103), the Shandong Key Research and Development Program (grant no. 2018GNC112008), and the Shandong "Double Tops” Program (grant no. SYL2017XTTD14).

Review statement. This paper was edited by Doina Pisla and reviewed by two anonymous referees.

\section{References}

Baskar, A. and Bandyopadhyay, S.: A homotopy-based method for the synthesis of defect-free mechanisms satisfying secondary design considerations, Mech. Mach. Theory, 133, 395-416, https://doi.org/10.1016/j.mechmachtheory.2018.12.002, 2019a.

Baskar, A. and Bandyopadhyay, S.: An algorithm to compute the finite roots of large systems of polynomial equations arising in kinematic synthesis, Mech. Mach. Theory, 133, 493-513, https://doi.org/10.1016/j.mechmachtheory.2018.12.004, $2019 \mathrm{~b}$.

Bai, S.: Geometric analysis of coupler-link mobility and circuits for planar four-bar linkages, Mech. Mach. Theory, 118, 53-64, https://doi.org/10.1016/j.mechmachtheory.2017.07.019, 2017.

Chanekar, P. V., Fenelon, M. A. A., and Ghosal, A.: Synthesis of adjustable spherical four-link mechanisms for approximate multi-path generation, Mech. Mach. Theory, 70, 538-552, https://doi.org/10.1016/j.mechmachtheory.2013.08.009, 2013.

Erdman, A. G. and Loftness P. E.: Synthesis of linkages for cataract surgery: storage, folding, and delivery of replacement intraocular lenses(IOLs), Mech. Mach. Theory, 40, 337-351, https://doi.org/10.1016/j.mechmachtheory.2004.07.006, 2005.

Filemon, E.: Useful ranges of centerpoint curves for design of crank-and-rocker linkages, Mech. Mach. Theory, 7, 47-53, https://doi.org/10.1016/0094-114X(72)90015-8, 1972.

Gupta, K. C. and Beloiu, A. S.: Branch and circuit defect elimination in spherical four-bar linkages, Mech. Mach. Theory, 33, 491-504, https://doi.org/10.1016/S0094-114X(97)00078-5, 1998.

Han, J. and Cao, Y.: Analytical synthesis methodology of RCCC linkages for the specified four poses, Mech. Mach. Theory, 133, 531-544, https://doi.org/10.1016/j.mechmachtheory.2018.12.005, 2019.

Han, J., Yang, T., Yin, L., and Qian, W.: Modern synthesis theory and method of linkage mechanism - analytical theory, domain solution method and software system, Higher Education Press, Beijing, China, 2013.

Martin, D. T. and Murray, A. P.: Developing classifications for synthesizing, refining, and animating planar mechanisms, ASME 2002 Design Engineering Technical Conferences and Computers and Information in Engineering Conference, 29 September-2 October 2002, Montreal, 2002.

McCarthy, J. M.: 21st Century Kinematics, Springer, London, 2013.
Murray, A. P. and Larochelle, P. M.: A classification scheme for planar 4R, spherical 4R, and spatial RCCC linkages to facilitate computer animation, 1998 ASME Design Engineering Technical Conferences, 13-16 September 1998, Atlanta, 1998.

Myszka, D. H., Murray, A. P., and Schmiedeler, J. P.: Assessing position order in rigid body guidance: an intuitive approach to fixed pivot selection, J. Mech. Design, 131, 0145021-0145025. https://doi.org/10.1115/1.3013851, 2009.

Ratcliffe, J. G.: Foundations of Hyperbolic Manifolds, Springer, New York, 2006.

Mendoza-Trejo, O., Cruz-Villar, C. A., Peón-Escalante, R., Zambrano-Arjona, M. A., and Peñuñuri, F.: Synthesis method for the spherical 4R mechanism with minimum center of mass acceleration, Mech. Mach. Theory, 93, 53-64, https://doi.org/10.1016/j.mechmachtheory.2015.04.015, 2015.

Ruth, D. A. and McCarthy, J. M.: The design of spherical 4R linkages for four specified orientations, Mech. Mach. Theory, 34, 677-692, https://doi.org/10.1016/S0094-114X(98)00048-2, 1999.

Shirazi, K. H.: Synthesis of linkages with four points of accuracy using Maple-V, Appl. Math. Comput., 164, 731-755, https://doi.org/10.1016/j.amc.2004.04.085, 2005.

Shirazi, K. H.: Computer modelling and geometric construction for four-point synthesis of $4 \mathrm{R}$ spherical linkages, Appl. Math. Model., 31, 1874-1888, https://doi.org/10.1016/j.apm.2006.06.013, 2007.

$\mathrm{Su}, \mathrm{H} . \quad$ J. and McCarthy, J. M.: The synthesis of an RPS serial chain to reach a given set of task positions, Mech. Mach. Theory, 40, 757-775, https://doi.org/10.1016/j.mechmachtheory.2005.01.007, 2005.

Sun, J., Chen, L., and Chu, J.: Motion generation of spherical four-bar mechanism using harmonic characteristic parameters, Mech. Mach. Theory, 95, 76-92, https://doi.org/10.1016/j.mechmachtheory.2015.08.020, 2016.

Tipparthi, H. and Larochelle, P.: Orientation order analysis of spherical four-bar mechanisms, J. Mech. Robot., 3, 04450110445014, https://doi.org/10.1115/1.4004898, 2011.

Waldron, K. J. and Strong, R. T.: Improved solutions of the branch and order problems of burmester linkage synthesis, Mecha. Mach. Theory, 13, 199-207, https://doi.org/10.1016/0094114X(78)90043-5, 1978.

Wang, J., Ting, K. L., and Xue, C.: Discriminant method for the mobility identification of single degree-of-freedom double-loop linkages, Mech. Mach. Theory, 45, 740-755, https://doi.org/10.1016/j.mechmachtheory.2009.12.004, 2010.

Zhao, P., Zhu, X., Li, L., Zi, B., and Ge, Q. J.: A novel motion synthesis approach with expandable solution space for planar linkages based on kinematicmapping, Mech. Mach. Theory, 105, 164-175, https://doi.org/10.1016/j.mechmachtheory.2016.06.021, 2016. 